\title{
Optimization of Battery Application for Wind Energy Storage
}

\author{
A. Danesh Shakib, G. Balzer \\ Institut of Electrical Power \& Energy \\ Technische Universität Darmstadt \\ Landgraf-Georg-Str. 4, 64283 Darmstadt (Germany) \\ Phone/Fax number:+49 06151 164259, e-mail: arefeh.danesh@eev.tu-darmstadt.de, gerd.balzer@eev.tu-darmstadt.de
}

\begin{abstract}
The largest disadvantage of wind energy is the lack of exact predictability and its fluctuations, which are causing problems in the power system operation. The consequence is that even though other energy sources can be saved by using wind energy, the short-term power fluctuation has to be secured by a conventional plant. A possible solution can be the storage of wind energy.

In this paper battery systems are analyzed as a backup for a 400 MW offshore wind farm with help of special developed calculation models. The goal is to reach small difference between forecasted and actual wind power and to increase the reliabilty of delivered wind energy using battery systems. For the determinations the properties of the battery system are considered and integrated into the calculation model. The overall target is to reduce the reserve energy requirement of the offshore wind farms.
\end{abstract}

\section{Key words}

Wind storage, wind, wind farm, modelling, reserve energy.

\section{Introduction}

In the last years, the planned capacity of large wind farms in Germany has been increased. Therefore, in the future the generation from wind energy will have a large penetration in the complete power generation [1].

Power output of the wind farms can have large fluctuations, which is a result of sudden wind speed changes [2]. In addition, the prediction of wind speeds is relatively difficult. Nowadays, the forecasting of wind speed is becoming better and better, but it is still not exact enough. This leads to larger need of reserve energy from conventional power plants.

A possible solution for wind power output and lower need of reserve energy is the storage of wind energy. There is a variety of storage technologies [3]. The application of battery systems is analyzed in this paper.

If the actual power output of the wind farm is higher than forecasted, the battery will be charged; on the other hand, if the power output is lower than the forecasted power, the battery will be discharged and covers the difference. Thereby, the wind power output into the system is always as planned, i.e. it has the forecasted amount. The actual charging state of battery is very important for its operational use.

\section{Wind Farm Operation with a Battery}

In order to keep the need of reserve energy in the system as low as possible, it is necessary to optimise the schedule of power plant operation, so that no considerable difference between electricity generation and load occurs. Due to already mentioned disadvantages of wind power, the planning of its production is much more difficult as for classical power plants. The reason is the stochastic character of the wind, which causes unforeseeable power fluctuations and increases considerably the requirements on the power control.

The principle operation of one wind farm- battery unit is shown at Fig. 1.

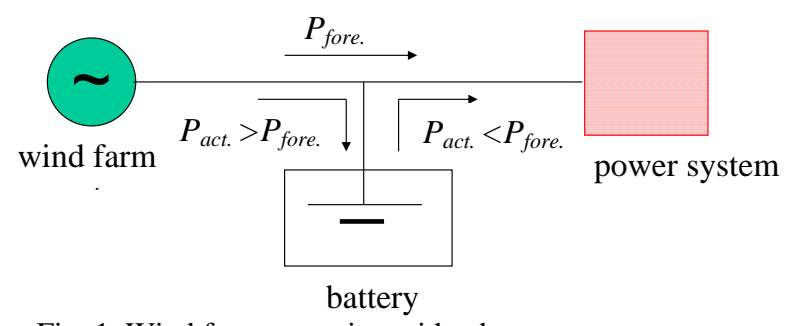

Fig. 1. Wind farm operation with a battery storage system.

The main task of the battery is to compensate the difference between the forecasted $\left(P_{\text {fore }}\right)$ und actual wind 
feeding $\left(P_{\text {act. }}\right)$ into the system. This leads to a higher reliableity of the wind farm.

Due to their good characteristics, the natrium-sulphur batteries (NAS) have been chosen as a backup of large wind farms [4]. The main features of the NAS batteries are large power and energy density, fast access time, large power capacity, large efficiency (up to 90\%) and relatively long life time ( 15 years or 2500 cycles). Due to large pulse power factor, they are able to feed higher power than the rated one into the system.

\section{Evaluation of Reserve Energy Economy Effect of the Battery}

In this paper a wind farm with $400 \mathrm{MW}$ installed capacity and a battarey system with 139 MW installed capacity were chosen. The forecasted and actual wind power values for 2006 are used from one of German transmission system operators E.ON [5].

A calculation model is developed for evaluation of reserve energy economy effect of the battery. This model is able to check efficiency of the battery application to compensate forecast errors, i.e. it calculates a reserve energy economy factor $\mathrm{S}$ as a function of battery size $\mathrm{P}_{\mathrm{B}}$, charge condition of battery $\mathrm{Ch}_{\mathrm{B}}$ and difference power values $\Delta P\left(t_{i}\right)$, where :

$$
\Delta P\left(t_{i}\right)=P_{\text {act. }}\left(t_{i}\right)-P_{\text {fore. }}\left(t_{i}\right)
$$

The model, which represents the evaluation process, is presented at Fig. 2.

At the beginning the initializing variables are being determined: charge condition of battery and number of samples $i$. Energy deficit or surplus $\Delta \mathrm{E}\left(t_{i}\right)$, as a consequence of forecast errors, is calculated for every time interval $t_{i}$ from the difference between actual and forecasted wind power $\Delta P\left(t_{i}\right)$, as:

$$
\Delta \mathrm{E}\left(t_{i}\right)=\Delta P\left(t_{i}\right) \cdot t_{\text {int }}
$$

By the calculation of the energy values, the program considers the efficiency factor of the battery and converter. Furthermore, the values of the battery's compensated energy $\mathrm{E}_{\text {compens. }}\left(t_{i}\right)$ and the battery's rest energy $\mathrm{E}_{\mathrm{rest}}\left(t_{i}\right)$ are calculated for every time interval.

The limitation of battery discharging and charging is considered by the calculation of the battery's rest energy. This value defines the new state of battery and is used for the next iteration.

Furthermore, for every iteration step $i$ the total energy, which the battery has to supply or store, and the total compensated energy by the battery are calculated. This process is repeated until the total number of samples $i_{\text {end }}$ is reached (e.g. for one year $i_{\text {end }}=35040$ ).

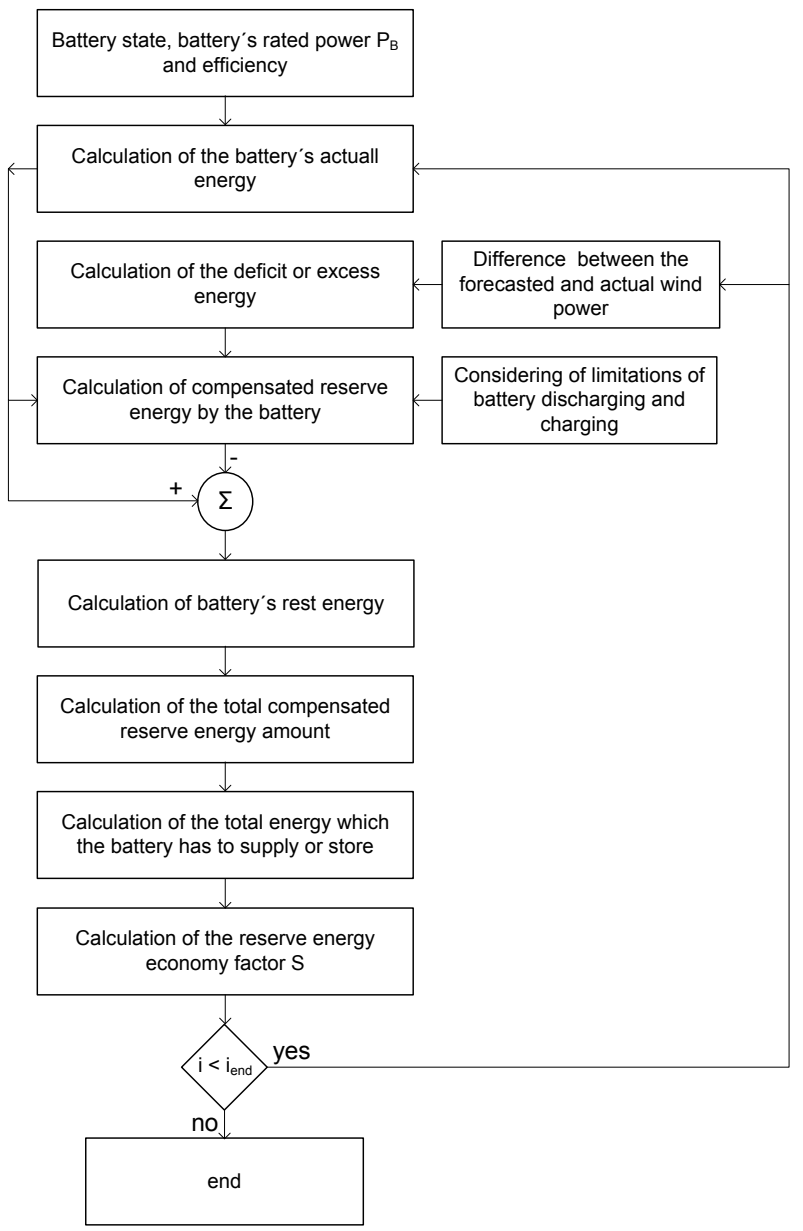

Fig. 2. The calculation process of reserve energy economy by a selected battery size [1]

Finally, the program calculates the reserve energy economy factor $\mathrm{S}$, which is defined as:

$$
S=\frac{\sum\left|\mathrm{E}_{\text {compens. }}\left(t_{i}\right)\right|}{\sum\left|\Delta \mathrm{E}\left(t_{i}\right)\right|}
$$

The reserve energy economy factor $\mathrm{S}$ shows how much reserve energy from conventional power plants can be saved by using batteries. The aim is to reach $100 \%$ compensation by battery $(\mathrm{S}=100 \%)$.

The calculations performed by the developed program at a representative offshore wind farm show the inadequate application of the batteries. Fig. 3 shows the calculated reserve energy economy factor, which can be achieved by the battery application with different sizes, for the analyzed wind farm. 


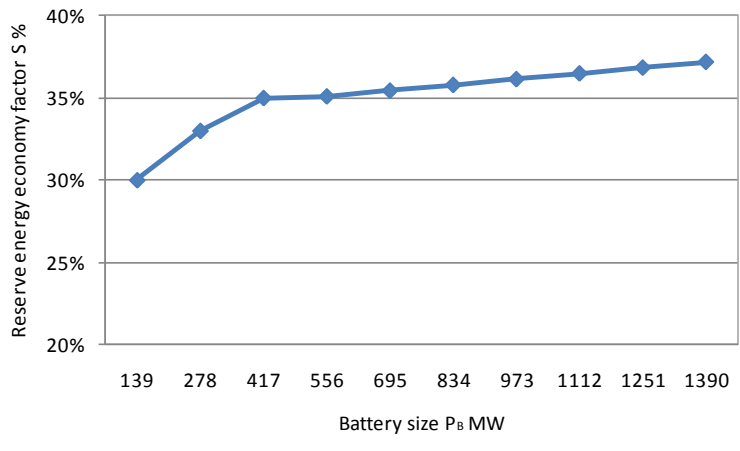

Fig. 3. Calculated reserve energy economy factor S vs. Battery size $\mathrm{P}_{\mathrm{B}}$.

It is difficult to reach a higher amount of $\mathrm{S}$ due to the fact that the sign of $\Delta P\left(t_{i}\right)$ does not change on a regular base which often leads to fully charging or discharging of the battery.

A higher reserve energy economy factor can be achieved with a larger battery, but the choice of a battery with higher rated power contradicts the economical aspects. The price for a NAS battery system amounts currently $2200 € / \mathrm{kW}$. This contains besides the battery costs, the costs of installation and a power conversion system PCS (AC/DC converter). The cost of PCS's adds up to 450 $€ / \mathrm{kW}$. Therefore in this work a battery cost of $1750 € / \mathrm{kW}$ is assumed [6].

\section{Optimization of the Battery Application}

There are several factors, which can help to reach a higher reserve energy economy factor of the battery. The first factor is the use of a larger battery, but as mentioned the choice of a battery with higher rated power contradicts the economical aspects. Fig. 3 shows that with a rated power of $417 \mathrm{MW}$ for the battery the reserve energy economy factor is increased at $5 \%$ but the using of larger battery as a backup system does not lead to significant changes of the factor $\mathrm{S}$.

The second factor is wind forecast accuracy. A lower forecast error leads to lower difference between forecasted and actual wind power and consequently to lower need of reserve energy. Fig. 4 shows the influence of forecast error on reserve energy of the battery for the two first January weeks.

With a better wind forecast, the wind farm energy is becoming closer to the forecasted wind energy. This leads to the increasing of reliability of wind energy supply. The calculation shows that with a lower forecast error $(4 \%)$ the reserve energy economy factor $\mathrm{S}$ increases at $7 \%$ for the two analyzed January weeks $\left(\left.\mathrm{S}\right|_{(\sigma \mathrm{error}=7.4 \%)}=\right.$ $\left.25 \% ;\left.\mathrm{S}\right|_{(\sigma \mathrm{error}=4 \%)}=32 \%\right)$. It can be seen that these both factors have not a significant impact of the optimization of the battery application.

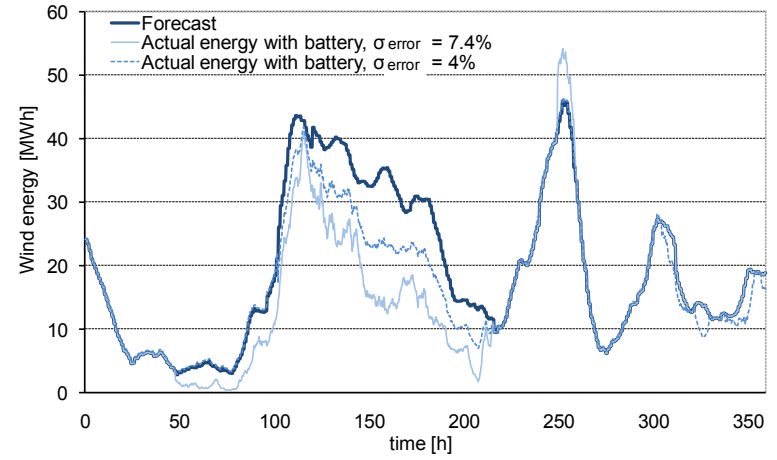

Fig. 4. Forecasted and actual wind energy for two different forecast errors.

Another factor is the charging state of the battery. The larger stored energy amount in the battery gives the possibility of compensating larger wind fluctuations. The battery charging state will be forecasted for the next forecast interval. If the battery is lower than half charged, the battery operator can offer less wind power than forecasted. If it is above half charged, he will offer more. Therefore, the fast completely discharging or charging of the batteries can be prevented. Thereby the forecast horizon is a very important factor. If the forecast horizon is shorter, more accurate data about wind speeds can be obtained. It is distinguished between day-ahead and intraday forecast [7]. Therewith, the use of the battery and its current state can be optimized better.

\section{Computation Model for the Optimization of the Battery Application}

A computation model which is automatically considering the charging state of the battery is developed. So the battery utilisation can be improved. The computation program works with the forecasted charging state of the battery. Relating to forecasted battery charging state, the battery operator offers more or less than forecasted wind feeding. Fig. 5 represents the principle of the calculation, wehre $\mathrm{m}$ is the number of time intervals of the forecasting horizon, $\eta_{\mathrm{B}}$ is the battery efiicienty, $\eta_{\mathrm{c}}$ is convertor efficienty, $\mathrm{Ch}_{\mathrm{B}}\left(\mathrm{t}_{\mathrm{o}}\right)$ is the actuall battery charging state at the determination beginning and $\mathrm{E}_{\max }$ is the maximal possible energy of the battery. 


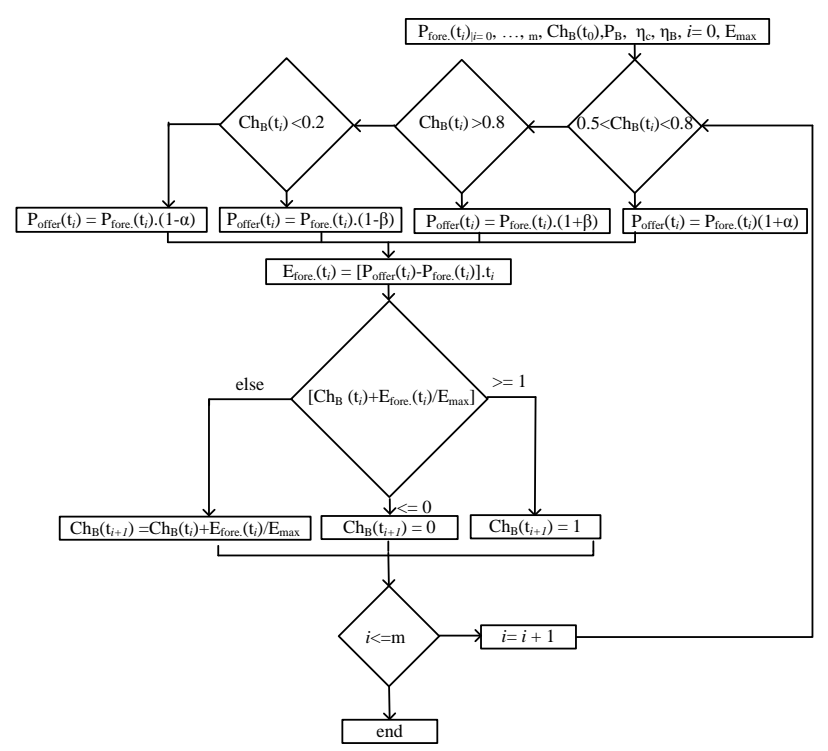

Fig. 5. Computation process for the wind feeding offers in the next forecasting horizon in consideration of the battery charging state (computation process A).

At the end of each forecasting horizon the data of the forecasted wind feeding for the next horizon and the actual wind feeding for the last horizon are known. With the help of the known data (the actual wind feeding and the offered wind feeding of the last horizon) is possible to calculate the actuall battery charging state at the beginning of the next forecasting horizon (Fig. 6) and use it as an input data for the computation process $\mathrm{A}$.

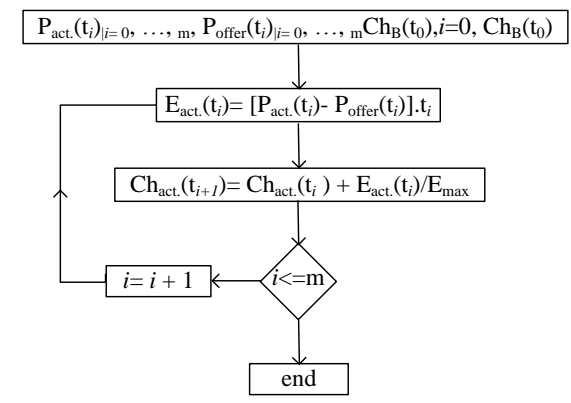

Fig. 6. Detremination of the actuell battery charging states for the last forecasting interval (computation process B).

Thereby the aim is to have very exact information about battery charging state for each forecasting intervals. The forecasting horizon is shorter the informations about battery charging state will be more exact.

For the calculation of the reserve energy economy factor following data are needed:

1) the actuall battery charging state for each forecasting interval (results of computation process B)Headers and Footers: None

2) the offered wind feeding for each forecasting interval (results of computation process A) and

3) the actuall wind power for each forecasting interval.

Fig. 7 shows the principle of the calculation.

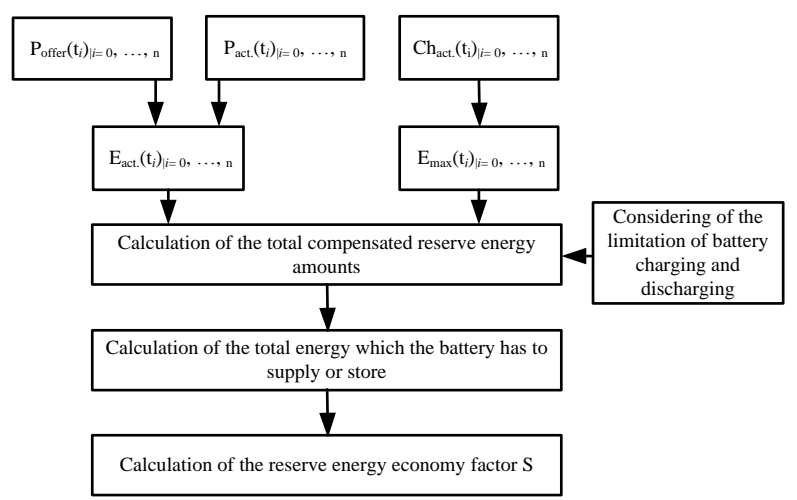

Fig. 7. Calculation of the reserve energy economy factor in consideration of the battery charging state.

\section{Computation Results}

For the analysis differnt assumptions are considered. Table I shows the analyzed forecasting horizons, battery sizes, assumed battery efficiency, assumed converter efficiency and assumed amount for $\alpha$ and $\beta$. For every forecasting horizon these factors can be varried and according to this the computation models determined automatically the relevant reserve energy economy factor.

Table I. The Assumed Factors for the Analysis

\begin{tabular}{|l|c|c|}
\hline Forecasting horizon & $24 \mathrm{~h}$ & $8 \mathrm{~h}$ \\
\hline Battery size & $139 \mathrm{MW}$ & $278 \mathrm{MW}$ \\
\hline Battery efficiency & \multicolumn{2}{|c|}{0,9} \\
\hline Convertor efficiency & \multicolumn{2}{|c|}{0,95} \\
\hline$\alpha$ & \multicolumn{2}{|c|}{$10 \%$} \\
\hline$\beta$ & $20 \%$ \\
\hline
\end{tabular}

The factors $\alpha$ and $\beta$ are selected random. Fig. 8 represented the calculated reserve energy economy factors by using of the calculation process demonstrated in Fig. 7.

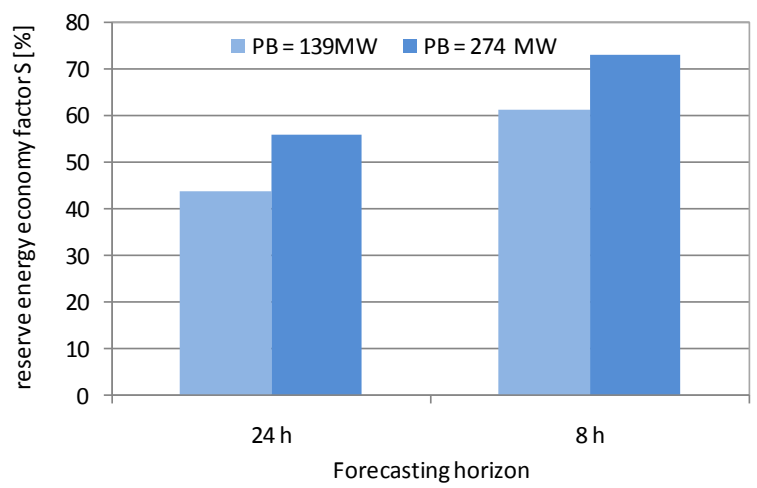

Fig. 8. Calculated $\mathrm{S}$ factors in consideration of battery charging state for two forecasting horizons and two battery sizes.

It is obvious that the time horizon and battery size play a decisive role. A significant improvement of the reserve energy economy factor has been obtained with a shorter time horizon and larger battery size. 
Furthermore, the factors $\alpha$ and $\beta$ has very important influence on the results of the calculation. The results show that it is absolutly possible to reach a higher compenastion of the forecasting errors with the use of other values for $\alpha$ and $\beta$ factors- Fig 9 .

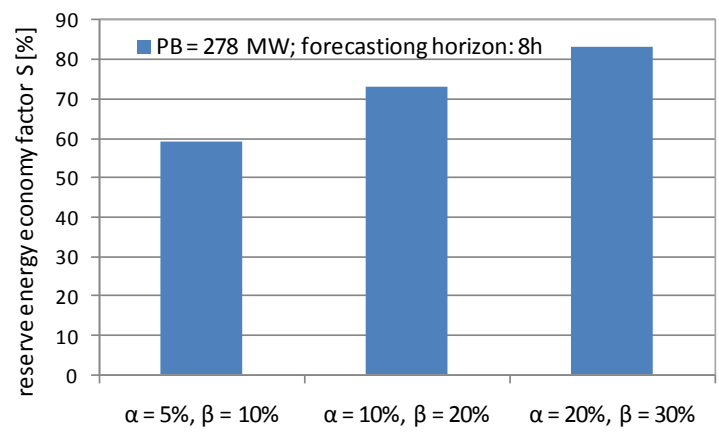

Fig. 9. Calculated $\mathrm{S}$ factors for three different $\alpha$ and $\beta$ factors.

The optimal sizing of the battery system under consideration of the different $\alpha$ and $\beta$ factors with a linear shape will be the subject for future studies.

\section{Conclusion}

The largest disadvantages of the wind energy are its weather dependency and high fluctuating character. This makes the wind power forecast very difficult. Therefore, the planning of the wind power production is much more difficult as for the classical power plants. The goal is to reach a possible small difference between forecasted and actual wind power. A possible solution for these problems can be the storage of wind energy.

The battery application has been analyzed as a backup for a $400 \mathrm{MW}$ wind farm for the compensation of power fluctuations with help of a developed calculation model. The results of analysis have shown that the use of $\mathrm{NaS}$ battery system to compensate a significant part of the power output fluctuation of the wind farm is possible. This leads to a considerably higher reliability of the wind power output and better reserve energy management. It has been shown that with consideration of the battery charging state, the battery application can be optimized. Furthermore, the battery size, the forecasting error and the forecasting horizon play an important role for the optimization of the battery application. From technical point of view the use of battery as a backup for the large wind farms is very meaningful, but the econimaical evaluation for the present and future application has to be analysed.

\section{Acknowledgement}

The autors gratefully thank "Stiftung Energieforschung Baden-Württemberg". Their financial support is highly appreciated.

\section{References}

[1] A.Danesh shakib, E. Spahić and G. Balzer, "Computation dimensioning of a sodium-sulphur battery as a backup of large wind farm", 16th Power System Computation Conference PSCC 2008, PS 39. Paper No. 481, Glasgow, Scotland, July 2008.

[2] E. Spahić and G. Balzer, "The Impact of the Wind Farm Size on the Power Output Fluctuations", European Wind Energy Conference EWEC 2006, Athena, Greek, Session B3, 27.02.-02.03.2006

[3] E. Spahić and G. Balzer, "The Application of Batteries as a Backup of Large Wind Farms", Large Scale Integration of Wind Power and Transmission Systems for Offshore Wind Farms, Paper No. 43, Delft, The Netherlands, October 2006

[4] M. Kamibayashi, D. K. Nichols and T. Oshima, "Development update of the NAS battery", in Proc. IEEE/PES Transmission and Distribution Conference and Exhibition, Yokohama, Japan, vol.3, pp 1664-1668, October 2002

[5] E.ON Netz [Online]. Available: http://www.eonnetz.com Euro-Wind: Windstromprognosen [Online]. Available: http://www.windprognose.

[6] E. Spahić, G.Balzer, W.Münch and B.Hellmich, "Wind energy storages - posibilities", PowerTech 2007, Lausanne, Switzerland, July.

[7] Euro-Wind: Windstromprognosen [Online]. Available: http://www.windprognose.de.

[8] M. Kamibayashi and K. Tanaka, "Recent Sodium Sulfur Battery Applications", in proc. IEEE/PES Transmission and Distribution Conference and Exposition, Atlanta, GA, USA, vol. 2, pp 1169-1173, February 2001. 